\title{
Feasibility online survey to estimate physical activity level among the students studying professional courses: a cross-sectional online survey
}

\author{
Bhumika Sudha', Asir John Samuel2, ${ }^{2 *}$, Kanimozhi Narkeesh' \\ 'Department of Musculoskeletal Physiotherapy, Maharishi Markandeshwar Institute of Physiotherapy and Rehabilitation, Maharishi Markandeshwar University, Haryana, \\ India \\ ${ }^{2}$ Department of Pediatric and Neonatal Physiotherapy, Maharishi Markandeshwar Institute of Physiotherapy and Rehabilitation, Maharishi Markandeshwar University, \\ Haryana, India
}

The aim of the study was to estimate the physical activity (PA) level among the professional college students in North India. One hundred three professional college students in the age group of 18-25 years were recruited by simple random sampling for this cross-sectional online survey. The survey was advertised on the social networking sites (Facebook, WhatsApp) through a link www.surveymonkey.com/r/MG588BY. A Short Form of International Physical Activity Questionnaire was used for this survey study. The questionnaire included total 8 questions on the basis of previous 7 days. The questionnaire consists of 3 main categories which were vigorous, moderate and high PA. Time spent in each activity level was multiplied with the metabolic equivalent of task (MET), which has previously set to 8.0 for vigorous activity, 4.0 for moderate activity, 3.3 for walking, and 1.5 for sitting. By multiplying MET with number of days and minutes performed weekly, amount of each activity level was calculated and measured as MET-min/wk. Further by adding MET minutes for each activity level, total MET-min/wk was calculated. Total number of 100 students participated in this study, and it was shown that all professional course students show different levels in PA. The total PA level among professional college students, which includes, physiotherapy, dental, medical, nursing, lab technician, pharmacy, management, law, engineering, were $434.4(0-7,866), 170.3$ $(0-1,129), 87.7(0-445), 102.8(0-180), 469$ (0-1,164), $0(0-0), 645(0-1,836)$, 337 (0-1,890), 396 (0-968) MET-min/wk respectively. PA levels among professional college students in North India have been established.

Keywords: College students, Exercise, Facebook, International physical activity questionnaire, Social media, WhatsApp

\section{INTRODUCTION}

Physical activity (PA) is any activity of body which involves energy consumption, by use of skeletal muscles hence involves bodily movements (Caspersen et al., 1985). Physical inactivity can leads to many health-related conditions like diabetes mellitus, hypertension, heart diseases etc. (Lee et al., 2012). Exercise is misunderstood with PA. Exercise is under PA, which is usually repetitive, structured, planned, and purposeful. Working, playing, active transportation, household works are part of PA as they involve bodily movements, and this is not precisely exercise (Caspersen et al., 1985). Life style of today young adults is more sedentary. Internet revolution, transportation revolution, food revolution, and mobile revolution have molded our life in every possible way (Mavrovouniotis, 2012). A number of changes have been there due to the advancement in the technology. Early humans move from place to place in search of food and shelter (Cordain et al., 1998). However, now, the cascade is riding on brain development as technology is making everything readily available to us.

The benefits of PA are to increase in growth and development,

\footnotetext{
${ }^{*}$ Corresponding author: Asir John Samuel (iD https://orcid.org/0000-0003-1747-0415 Department of Pediatric and Neonatal Physiotherapy, Maharishi Markandeshwar Institute of Physiotherapy and Rehabilitation, Maharishi Markandeshwar University, Mullana-133207, Haryana, India

Tel: +91-1731274522/9481939806,

E-mail: asirjohnsamuel@mmumullana.org, asir.j.samuel@gmail.com

Received: October 5, 2017 / Accepted: January 11, 2018
}

This is an Open Access article distributed under the terms of the Creative Commons Attribution Non-Commercial License (http://creativecommons.org/licenses/by-nc/4.0/) which permits unrestricted non-commercial use, distribution, and reproduction in any medium, provided the original work is properly cited. 
prevents aging, helps to strengthen the muscles, improves cardiovascular endurance, helps to maintain healthy body weight, boosts immune system, helps to prevent diseases such as cardiovascular disease, type-2 diabetes mellitus, obesity, helps to prevent stress and depression, enhances mood, promotes sleep, reduces blood sugar level, boosts energy, regulate blood pressure, and improve social well-being (Sothern et al., 1999).

Various studies were performed on university students to highlight the importance of PA among young adults. A recent research reported that among the university students, women perform less vigorous activity than men (Fagaras et al., 2015). Another researcher reported that fourth year medical college students were engaged in higher levels of PA than average young adults of similar age (Holtz et al., 2013). A cross-sectional study was performed on students using International Physical Activity Questionnairre-Short Form (IPAQ-SF) to measure PA reported that $11.3 \%$ of participants were physically inactive, $52 \%$ had moderate activity, 36.7\% had high PA levels (El-Gilany et al., 2011). In describing the PA behaviors among medical students in Southern Thailand, researcher reported approximately half of the participants (49.5\%) were physically active. More than half of the medical students have insufficient PA because of study-related activities and overtime shift work (Wattanapisit et al., 2016).

A cross-sectional study was executed on 409 students to assess the PA habits among medical students explored the major obstacles preventing the students from PA were laziness, lack of time, studying and lack of interest (Al-Drees et al., 2016). A survey on 250 medical students reported that they were engaged in physical exercise prior to their admission into medical college (Al-Asousi and El-Sabban, 2016). A research work was done to determine the prevalence and patterns of PA among young adults. Forty-one point three percent (41.3\%) of students showed high levels of PA, $43.2 \%$ and $15.4 \%$ of students showed moderate level and low level of PA respectively. Eighty-four point six percent (84.6\%) were engaged in work related activity and $80.7 \%$ showed transport related activity. Domestic and gardening PA represented $63.7 \%$ of individual's total activity and $67.2 \%$ of students showed leisure time activity. The average time spent in sitting was $7.1 \mathrm{hr} /$ day. The median of the total PA for the whole sample was 39.13 metabolic equivalent of task (MET)-hr/wk and 18.10 MET-hr/wk for work, 4.40 MET-hr/wk for transportation, 2.60 MET-hr/wk for domestic and gardening and $4 \mathrm{MET}$-hr/wk for leisure time activity. There was significant gender difference observed with women having low PA (Padmapriya et al., 2013). The study by using the IPAQ-SF demonstrated that adolescents with idiopathic scoliosis showed similar levels of self-reported PA as individuals without idiopathic scoliosis (Diarbakerli et al., 2016).

During college time, students become more sedentary and their PA gets reduced. PA forms an integral aspect of day-to-day living. But young people at recent days, spend most of their time by using mobile phones and surfing the internet without any PA. Since a large part of their time is spent on this activity, and they develop conditions like, stress, insomnia, neck pain, back pain, obesity, anxiety, depression, social phobia, lack of appetite, cardiovascular diseases, etc. With advancement of technology, e-books are becoming part of studies. There is no time for youth to play or do any PA. In present scenario youth is more and more getting into electronical devices like mobiles rather than indulging in outdoor games. This is dangerous because it is inviting not only wrist and cervical problems but also increasing risk factors of ischemic heart diseases, diabetes mellitus, obesity and many more. PA forms an integral part of everyday life among the professional college students. But the level of PA among the professional college students of North India is not evaluated yet. Hence, we intend to explore the PA level among north Indian professional college students.

\section{MATERIALS AND METHODS}

The study protocol was approved by the institute students project committee (MMIPR/SPC/2017/14) and the study was done strictly in accordance with the guidelines of Helsinki declaration, revised 2013 (World Medical Association, 2013). One hundred three professional college students in the age groups, 18 to 25 years were selected by simple random sampling for this cross sectional survey study. The study was conducted using a link shared on social networking sites through a validated questionnaire, IPAQ-SF. The survey was advertised on social media (Facebook [social media] and WhatsApp [an android based messaging mobile application]) through a link (www.surveymonkey.com/r/MG588BY) which included the project description. We have not sent through email as it was not a first option to reach large number of college students. Individual accessing these apps was invited to participate in the study if they met the inclusion criteria. The survey was open for the participants studying in professional college in north India.

The online survey was selected because it is cost effective, time saving, and easily accessible. The survey was administered using the online survey portal, Survey Monkey (Online survey services, San Mateo, CA, USA). The survey took approximately $10 \mathrm{~min}$ to complete. A survey provides a standardized approach allowing 
uniformity of questions to all the participants. Data collection was based on a validated questionnaire, IPAQ-SF that was designed to fall into three main categories, which are vigorous, moderate and mild PA. The questionnaire is filled up by students of medical and nonmedical courses. Any inquiries about the meaning of certain question were provided by the researcher through phone contact. If the student attempted the survey, then it was considered that they have provided consent to participate in the study.

The questionnaire included total eight questions. In that questionnaire there are three main categories, which are vigorous, moderate, and mild PA. Under vigorous PA category, there are 2 question, which are listed about PA like heavy lifting, aerobics, fast bicycling, heavy lifting, under moderate PA category, there are two questions, which are listed about PA like lifting light weights, doubles tennis, under mild PA category, there are two questions, which are listed about walking, sitting at a regular place.

Time spent in each activity level were multiplied with MET which have previously set to 8.0 for vigorous activity, 4.0 for moderate activity, 3.3 for walking, and 1.5 for sitting. By multiplying the MET values with number of days and minutes performed weekly, amount of each activity level was calculated and represented as MET-min/wk. Further, by adding MET-min/wk for each activity level, Total PA level among the students studying professional courses were calculated.

Normality of the collected data was analyzed by KolmogorovSmirnov test. As the data does not follow normal distribution, we have expressed the central tendency and dispersion of the data as mean with $95 \%$ confidence interval and median with interquar- tile range. The significance difference of PA among groups, where demonstrated by Kruskal-Wallis test. For all the analysis, the levels of significance were set 0.05 to minimize type 1 error. The above statistical analysis was performed using the statistical software, SPSS ver. 16.0 (SPSS Inc., Chicago, IL, USA).

\section{RESULTS}

Among 103 online surveys, three survey reports were incomplete. Hence, the study is based on only 100 survey reports. Details of the professional college students participated in this study were given in Table 1. Overall PA level was described in Fig. 1. Individual PA level, like, vigorous, moderate, walking, sitting among the professional college students were portrayed in Figs. 2-6. The total PA level among professional college students, which includes, physiotherapy, dental, medical, nursing, lab tech-

Table 1. Number of professional college students in each stream participated $(\mathrm{n}=100)$

\begin{tabular}{clc}
\hline Student No. & Professional course & No. of students participated \\
\hline 1 & Physiotherapy & 30 \\
2 & Dental & 10 \\
3 & Medical & 7 \\
4 & Nursing & 4 \\
5 & Lab technician & 6 \\
6 & Pharmacy & 2 \\
7 & Management & 3 \\
8 & Law & 6 \\
9 & Engineering & 9 \\
10 & Not specified & 23 \\
\hline
\end{tabular}

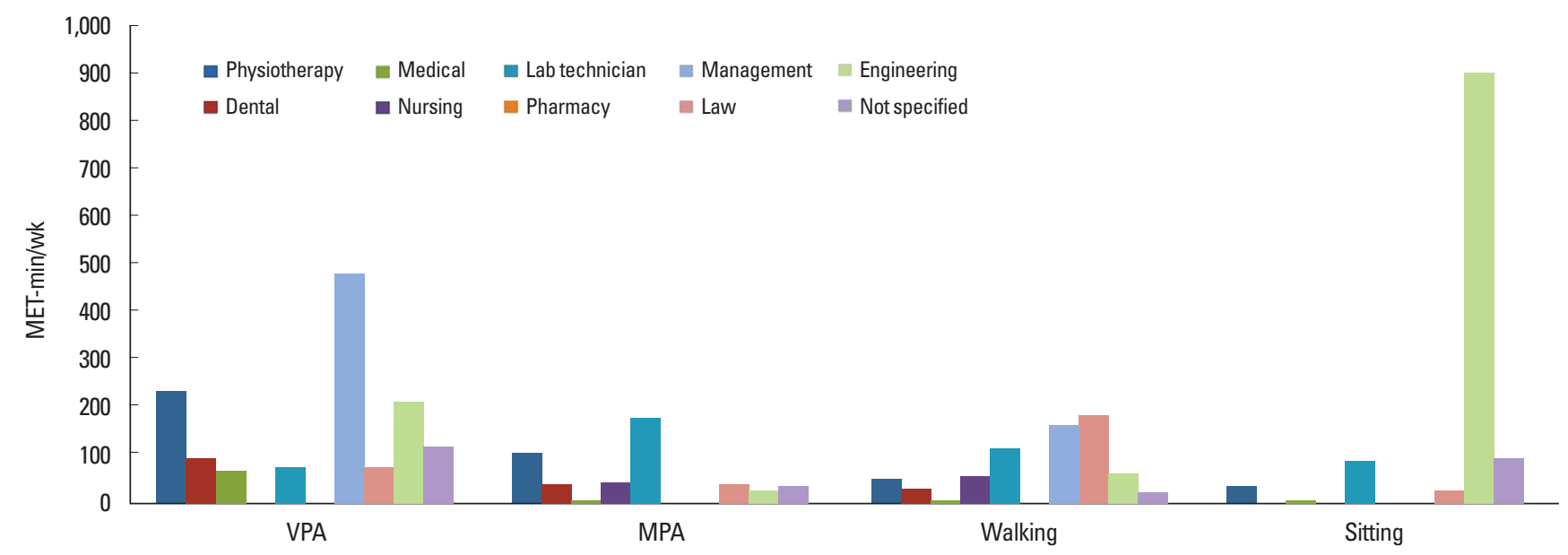

Fig. 1. Mean physical activity (PA) level (expressed in MET-min/wk) among the professional college students in North India. MET, metabolic equivalent of task; VPA, vigorous physical activity; MPA, moderate physical activity. 


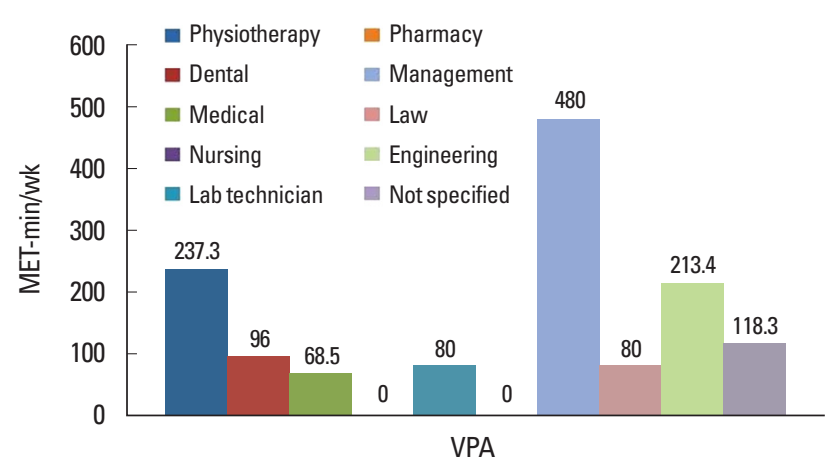

Fig. 2. Mean vigorous physical activity (VPA) level (expressed in MET-min/wk) among the professional college students in North India. MET, metabolic equivalent of task.

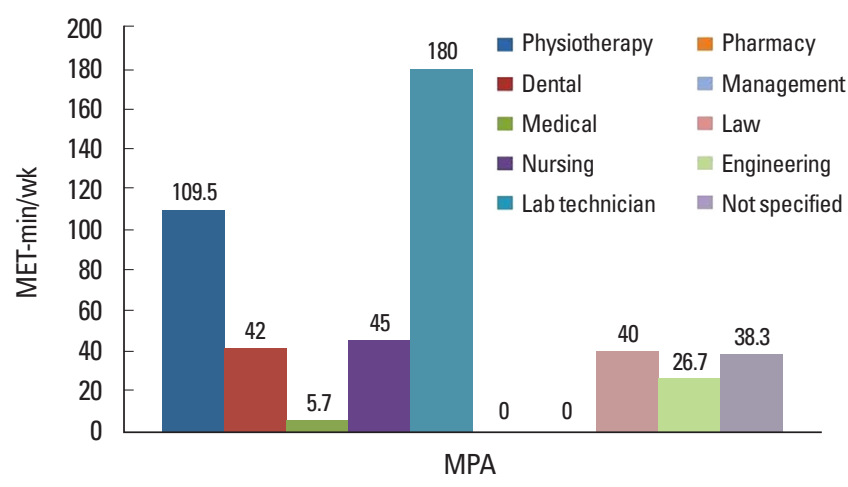

Fig. 3. Mean moderate physical activity (MPA) level (expressed in MET-min/ wk) among the professional college students in North India. MET, metabolic equivalent of task.

nician, pharmacy, management, law, engineering, were 434.4 ( 0 7,866), $170.3(0-1,129), 87.7(0-445), 102.8(0-180), 469(0-$ 1,164), 0 (0-0), $645(0-1,836), 337(0-1,890), 396$ (0-968) MET-min/wk respectively.

\section{DISCUSSION}

This study is a cross-sectional online survey. Online survey is one of the most widely utilized survey methods. An online survey is the systematic gathering of the data from the respondents. The Internet is a vast virtual world that connects all kind of people from around the globe. Hence, a survey that requires hundred or more respondents could be conducted through the internet. Online survey facilitates low-cost and fast data collection from the target population. Sending online questionnaire is more affordable than the face-to-face method. Online survey provides the highest level of convenience for the respondents because they can answer the questionnaire according to their chosen time. The survey took

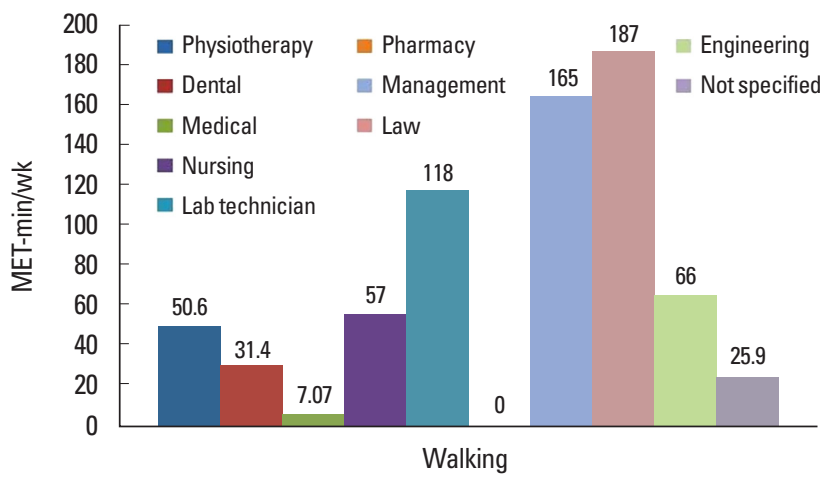

Fig. 4. Mean walking physical activity level (expressed in MET-min/wk) among the professional college students in North India. MET, metabolic equivalent of task.

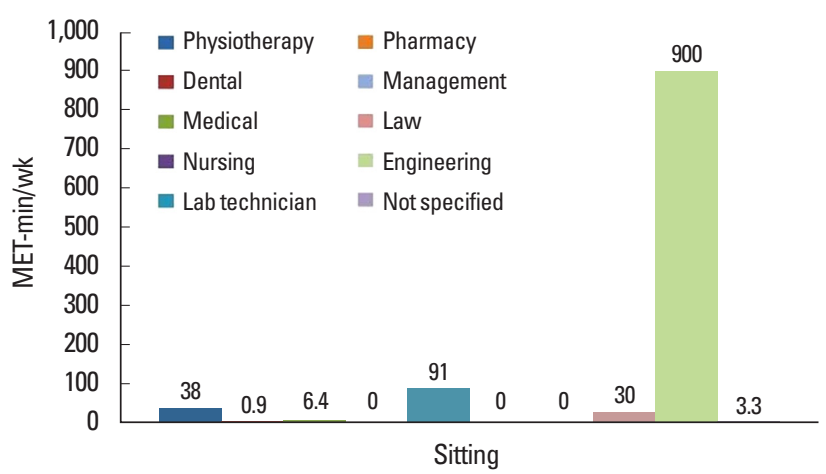

Fig. 5. Mean sitting physical activity level (expressed in MET-min/wk) among the professional college students in North India. MET, metabolic equivalent of task.

approximately $10 \mathrm{~min}$ to complete. In the present online survey study, 103 professional college students were included through a link sent on social networking sites, Facebook and WhatsApp. IPAQ-SF was used as it was designed to fall into three main categories, which are vigorous, moderate and mild PA.

The findings of the study revealed that management students were doing more vigorous activity (645 MET-min/wk) whereas lab technician students are more involved in moderate PA (469 MET-min/wk), law students are the ones who are walking the most (337 MET-min/wk) and engineering students are known to sit more hours (396 MET-min/wk) than other professional course students.

One similar study was conducted by Diarbakerli et al. (2016), in which level of PA were measured in adolescents with and without idiopathic scoliosis. Two hundred thirty-nine adolescents with idiopathic scoliosis and 58 adolescents without idiopathic scoliosis were recruited. The findings of this study did not show any difference in PA level in adolescents with idiopathic scoliosis compared 


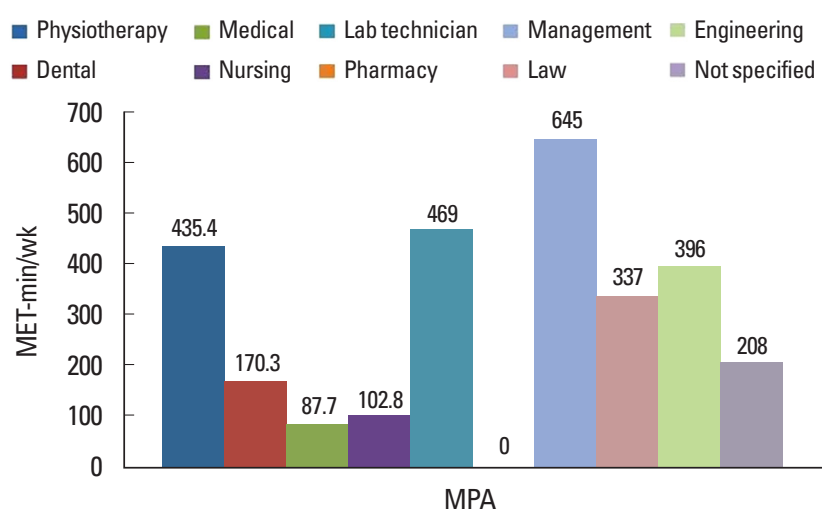

Fig. 6. Mean total physical activity level (expressed in MET-min/wk) among the professional college students in North India. MET, metabolic equivalent of task; MPA, moderate physical activity.

to individuals without idiopathic scoliosis. Median MET-min/wk for individuals without idiopathic scoliosis was 2,120 MET-min/ wk and Median MET-min/wk for our study is 606.3 MET-min/ wk, which is lower than their study.

Another study was done by Fagaras et al. (2015), in which level of PA of university students were measured. Three hundred thirty-three students participated in this study were asked to respond to an IPAQ categorized as: (a) vigorous activity; (b) moderate activity; (c) walking, and (d) sitting in last seven days. The MET value for their study is $1,395.18 \mathrm{MET}$ min/week for walking, 1,111.12 MET min/wk for moderate PA, and 2,867.34 MET $\mathrm{min} / \mathrm{wk}$ for vigorous PA and the MET values for our study are $187 \mathrm{MET}$ min/wk for walking (law students), $180 \mathrm{MET}$ min/wk for moderate PA (laboratory technician students), and 480 MET $\mathrm{min} / \mathrm{wk}$ for vigorous PA (management students) which is lower than their study.

El-Gilany et al. (2011) conducted a research to describe the pattern of PA on 1,708 students. The IPAQ was used to measure PA. The findings showed that $42 \%$ of college students participated in vigorous activity at least 3 times a week, while an additional $20 \%$ participated in moderate activity. The total mean value for their study was 3,133.0 MET-min/wk and the mean value for our study is 2,828.8 MET-min/wk which is lower than their study. The barriers to PA in their study was lack of interest in PA, lack of time, parents giving academic success priority over exercise, lack of convenient facilities and health conditions which might be similar in our study.

Lobelo et al. (2009) conducted a study to describe PA habits of physicians and medical students influence their counseling practices. This study found comparable results with our study that medical professionals are a credible and respected source of health-related information would provide ongoing preventive counseling feedback and follow-up. The MET value for medical students in our study is $87.7 \mathrm{MET}-\mathrm{min} / \mathrm{wk}$ and the MET value for their study was 105 MET-min/wk, which is higher than our study.

Another similar study conducted by Wattanapisit et al. (2016), to describe PA among medical students. A total of 279 medical students participated in the study. The study showed consistent result with our study that. Approximately half of the participants were physically active. The median value for their study was 540 MET-min/wk (range, 0-5,640 MET-min/wk) and the mean value for our study is $87.7(0-445) \mathrm{MET}-\mathrm{min} / \mathrm{wk}$, which is lower than their study.

When compared to other literature on PA, the professional college students of North India have low PA. Their PA level could be enhanced by adding PA activity classes in study protocol and social support from friends and family, by organizing health promotion programme. As the study is an online survey, we were able to attain wider reachability and visibility, eco-friendly (papers were not used), cost effective, minimum time consuming and able to respond by participant at the time of their convenience. These were the strength of the study. Though the study is not free from limitations like unequal distribution of professional college students, limited sample size, and limited to the student who use social media which might not represent the actual PA level among the students. However, this is the first among the study using online survey and social media in reporting the PA level. The feasibility of online survey has been verified.

The study highlights the PA levels among professional college students in North India. PA levels among professional college students in North India have been established and their PA level is low when compared to other of similar age groups. The feasibility of online survey in reporting PA level among professional college students has been verified.

\section{CONFLICT OF INTEREST}

No potential conflict of interest relevant to this article was reported.

\section{ACKNOWLEDGMENTS}

Special thanks to Dr. Vencita Priyanka Aranha, MPT, Assistant Professor from Maharishi Markandeshwar Institute of Physiotherapy and Rehabilitation, Maharishi Markandeshwar University, in 
manuscript technical editing. This research forms the partial fulfillment of the internship completion programme of the structured Bachelor of Physiotherapy (BPT) curriculum.

\section{REFERENCES}

Al-Asousi M, El-Sabban, F. Physical activity among preclinical medical students at The University of Malaya , Malaysia. J Nutr Health Food Sci 2016;4:1-8.

Al-Drees A, Abdulghani H, Irshad M, Baqays AA, Al-Zhrani AA, Alshammari SA, Alturki NI. Physical activity and academic achievement among the medical students: a cross-sectional study. Med Teach 2016;38 Suppl 1:S66-S72.

Caspersen CJ, Powell KE, Christenson GM. Physical activity, exercise, and physical fitness: definitions and distinctions for health-related research. Public Health Rep 1985;100:126-131.

Cordain L, Gotshall RW, Eaton SB, Eaton SB 3rd. Physical activity, energy expenditure and fitness: an evolutionary perspective. Int J Sports Med 1998;19:328-335

Diarbakerli E, Grauers A, Möller H, Abbott A, Gerdhem P. Adolescents with and without idiopathic scoliosis have similar self-reported level of physical activity: a cross-sectional study. Scoliosis Spinal Disord 2016;11:17.

El-Gilany AH, Badawi K, El-Khawaga G, Awadalla N. Physical activity profile of students in Mansoura University, Egypt. East Mediterr Health J 2011;17:694-702.

Fagaras SP, Radu LE, Vanvu G. The level of physical activity of university students. Procedia - Soc Behav Sci 2015;197:1454-1457.

Holtz KA, Kokotilo KJ, Fitzgerald BE, Frank E. Exercise behaviour and attitudes among fourth-year medical students at the University of British Columbia. Can Fam Physician 2013;59:e26-e32.

Lee IM, Shiroma EJ, Lobelo F, Puska P, Blair SN, Katzmarzyk PT; Lancet Physical Activity Series Working Group. Effect of physical inactivity on major non-communicable diseases worldwide: an analysis of burden of disease and life expectancy. Lancet 2012;380:219-229.

Lobelo F, Duperly J, Frank E. Physical activity habits of doctors and medical students influence their counselling practices. Br J Sports Med 2009;43:89-92.

Mavrovouniotis F. Inactivity in childhood and adolescence: a modern lifestyle associated with adverse health consequences. Sport Sci Rev 2012;21:75-99.

Padmapriya K, Krishna P, Rasu T. Prevalence and patterns of physical activity among medical students in Bangalore, India. Electron Physician 2013;5:606-610.

Sothern MS, Loftin M, Suskind RM, Udall JN, Blecker U. The health benefits of physical activity in children and adolescents: implications for chronic disease prevention. Eur J Pediatr 1999;158:271-274.

Wattanapisit A, Fungthongcharoen K, Saengow U, Vijitpongjinda S. Physical activity among medical students in Southern Thailand: a mixed methods study. BMJ Open 2016;6:e13479.

World Medical Association. World Medical Association Declaration of Helsinki: ethical principles for medical research involving human subjects. JAMA 2013;310:2191-2194. 